\title{
Proyecto de vida y su relación con valores, auto-concepto y apoyo social percibido en estudiantes de secundaria
}

\section{Rebeca Vargas}

Universidad de Iberoamérica

\section{Resumen}

La siguiente investigación fue realizada con el fin de conocer cuáles factores se encuentran relacionados con el grado de claridad que poseen los adolescentes de décimo año con respecto a su Proyecto de Vida. Para esto se realizó un estudio cuantitativo no experimental con una población total $(\mathrm{N}=120)$ de estudiantes de décimo año de dos Colegios ubicados en Heredia; el Colegio Manuel Benavides y el Colegio Bilingüe Santa Inés, una institución pública y privada respectivamente. Para la recolección de datos se utilizó un instrumento de medición tipo Likert y se evaluaron las escalas de Proyecto de Vida ( $\alpha:$.71), Valores ( $\alpha: .78)$, Autoconcepto ( $\alpha: .72)$ y Apoyo Social Percibido ( $\alpha$ : $.93)$.

Los resultados comprueban que sí existe una relación positiva con todas las variables estudiadas y la variable Proyecto de Vida: Valores $(r=.41 ; p<.001)$, Autoconcepto $(r=.37 ; p<.001)$, Apoyo Social $(r=.26 ; p=.005)$. Además se obtiene como mayor predictor el Autoconcepto ( $\beta .250$ $p=.005)$ seguido por la variable Valores $(\beta .243 p=.009)$ y por último el Sexo $(\beta .225 p=.009)$. Sin embargo no se encuentra relación entre el Proyecto de Vida y el tipo de institución a la que asistan los adolescentes.

Palabras Clave: Proyecto de vida, adolescencia, valores morales, autoconcepto, apoyo social

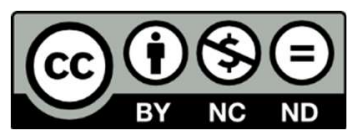




\section{Introducción}

La fase adolescente tiende a ser estudiada bajo un enfoque más centrado en las problemáticas que se presenta (tanto sociales como personales), dejando de lado aspectos positivos y la manera en que podemos resaltar y preservar estos (Escudero, 2011). En ese sentido, la presente investigación se concentra en brindar un enfoque que preste más atención a la preservación y promoción del bienestar que puedan impulsar la calidad de vida a futuro, más allá de apuntar solamente al tratamiento de ciertas condiciones o situaciones difíciles.

Se trata de un periodo de la vida lleno de cambios y decisiones complejas que constituye un reto en el que estos deben empezar a seleccionar y a prepararse para su inserción en el mundo laboral a la vez que adquieren conocimiento adecuado para desempeñarse en su sociedad respectiva, lo que implica establecer y mantener relaciones a la vez que se independizan de manera progresiva (Center of Population Options, 1990). Domínguez (2002) define proyecto de vida como aquel

(...) Sistema de objetivos mediatos vinculados a las principales esferas de realización del joven, entre las que pueden encontrarse la familia, la profesión, su autorrealización, etc. Tiene como importante sostén el conjunto de valores que se estructuran como contenidos de su concepción del mundo y que también forman parte de su identidad personal (p.165).

Salazar, 2009, afirma que de trabajar cada uno de los componentes del proyecto de vida desde la secundaria, este no se vería interrumpido en ningún momento y a la vez se reducirían patologías sociales como ansiedad, depresión, baja autoestima, etc, que son comunes en esta edad.

En cuanto a estudios nacionales, Álvarez (2005) concluye que el taller realizado sobre proyecto de vida logra crear confianza en los participantes y darles la sensación de que ellos pueden lograr lo que se propongan a pesar de que puedan presentar dificultades mayores.

\section{psco


Dicho taller buscaba orientar e informar a adolescentes que se encuentren internados en el Hogar Pétalos de Rosa del PANI, ubicado en Hatillo 2. El estudio se conformó de siete actividades en las que pretendió brindar información a los jóvenes y lograr que estos se cuestionaran quiénes son y qué esperan de sus vidas, además de tratar temas como la motivación propia, más allá de la falta de apoyo familiar. La autora hace énfasis en cómo una gran parte del aprendizaje no se obtiene sólo en la educación formal, sino que es un proceso que se da en el diario vivir, ya sea por observación, reforzamiento o castigo.

Propiamente sobre el proyecto de vida, afirma que: "Es precisamente en la adolescencia, aproximadamente entre los 15 y 19 años, en nuestra cultura, que emerge la necesidad de proyectarse laboralmente, y por ello se delinean con más claridad los conflictos relativos al acceso al mundo adulto en términos ocupacionales" (Álvarez, 2005, p. 204).

Erickson (1968) y Santrock (2002) citados por Chaves (2005), indican que "el aspecto más importante en la adolescencia es el desarrollo de la identidad, pues en esta etapa se buscan respuestas (...) estos temas no se consideran durante la niñez, pero emergen durante los años de preparatoria y universidad como temas comunes y prácticamente universales" (p.35).

Esta autora afirma que las relaciones interpersonales en la adolescencia poseen una gran influencia en la construcción de la identidad, toma de decisiones y la importancia del apoyo social en esta área, además de cumplir con una función importante en la resolución de conflictos y en la formación de habilidades para la vida.

Müller (1994, citado por Artavia, 2011) en cuanto al concepto de Orientación Vocacional lo define como "campo de trabajo preventivo de adolescentes, jóvenes y adultos, con conflictos o dudas para elegir estudios y ocupaciones, con deseos de cambiar los mismos, o que presentan situaciones en la vida en las cuales su decisión ha fracasado" (p.45). 
En dicho estudio se hace un abordaje del Proyecto de Vida desde 4 variables principales: identidad, eventos del ciclo de vida, autoestima, estructura y dinámica del medio ambiente.

En cuanto a investigaciones internacionales, Salazar (2009) realiza una propuesta para los adolescentes sobre cómo planear su proyecto de vida:

La planeación de vida estará fundamentada por una actitud que se caracterizará por el esfuerzo, arte y disciplina de conocerse a sí mismo, de detectar las fuerzas y debilidades y proyectarse autodirigiendo su propio destino; es el funcionamiento pleno de las capacidades, motivaciones y adjetivo de la vida personal y familiar, social y de trabajo (Salazar, 2009, p 9.)

Center of Population Options (1990) realizó pruebas de campo para determinar la eficacia y utilidad de la Educación para la planeación de la vida en Latinoamérica. Midieron cambios de actitud en los adolescentes después de recibir el curso "educación para la planeación de la vida". Los resultados arrojaron cambios de actitudes y conocimientos sobre la protección en las relaciones sexuales, la importancia de la comunicación personal y algunos puntos relevantes sobre cómo conseguir trabajo.

Damon, Menon y Cotton (2003) definen propósito de la siguiente manera: "El propósito es una parte de la propia búsqueda de significado personal, pero también posee un componente externo, el deseo de hacer una diferencia en el mundo, contribuir a asuntos más allá de los propios” (p.121).

Basados en una detallada revisión bibliográfica, los autores llegan a la conclusión de que los adolescentes hoy en día no poseen las mismas categorías de propósitos que se tenían tradicionalmente como: familia, fe, comunidad, país y trabajo, esto podría deberse a que los universitarios y los medios de comunicación ya no apoyan estos propósitos. Sin embargo, es necesario realizar una investigación

\section{UT


para descubrir si existen nuevas categorías que reemplacen las tradicionales o cuáles propósitos son los que los adolescentes persiguen actualmente.

Otros estudios han relacionado el proyecto de vida con problemáticas que viven los adolescentes actualmente. Este es el caso de la investigación realizada por Acosta y Gastelo (2012) en la que relacionan los embarazos adolescentes con el proyecto de vida. Ellas plantean analizar las condiciones de las madres y estudiantes embarazadas en el colegio y cómo esto repercute en el proyecto de vida de las mismas. La investigación se realizó con adolescentes embarazadas y madres adolescentes de 15 liceos públicos de Cumaná, Estado Sucre.

Los resultados obtenidos durante la investigación concluyen que la mayoría de las adolescentes con las que se trabajó no logran alcanzar un grado educativo superior por lo que no logran completar su proyecto de vida, teniendo que dedicarse a otras labores como el cuido del menor, además de trabajar para lograr satisfacer las necesidades de este.

Roebken (2007), realiza un estudio donde examina la relación que existe entre la orientación de metas de los estudiantes y la satisfacción estudiantil, el compromiso académico y el logro. Se utilizaron 2309 estudiantes de una Universidad de California para conocer la relación entre los tipos de metas de los estudiantes y su comportamiento y resultados académicos. Los resultados demuestran que los estudiantes que persiguen sus metas están más satisfechos con su experiencia académica, muestran un mayor grado de compromiso y obtienen mejores calificaciones.

Domínguez (2002), caracteriza elementos como identidad, valores y proyecto de vida ya que considera que son importantes para el desarrollo de una personalidad sana y madura y como la enseñanza y educación puede tener influencia en el desarrollo de estas. Indica que los valores "forman parte de la subjetividad humana y aparecen como formaciones complejas de la personalidad en la edad juvenil con el surgimiento de la concepción del mundo" (p.164). 
Orviz, Sánchez y Valdés (2011), realizan un estudio que busca entender las razones del por qué los jóvenes toman distintas decisiones sobre sus proyectos de vida, además de comprender el aumento de la deserción escolar y de la evasión de una visión a futuro en el ámbito laboral. El objetivo de dicha investigación es "Conocer la visión a futuro de un adolescente que cursa actualmente la secundaria, en la elección de su ocupación en la vida adulta". Para esto se eligió un adolescente de 15 años que cursaba el tercer año de secundaria.

Las autoras concluyen que es importante el apoyo de los centros educativos para promover el pensamiento en los jóvenes sobre qué desean para el futuro y los impulsen a conocer a fondo sobre profesiones y ocupaciones. Plantean que una manera de apoyar a los jóvenes para tomar decisiones sobre su futuro laboral puede ser convivir un día con una persona que se dedique a la profesión que han considerado.

Freite, Vesga y Villanueva (2011) tienen como objetivo "fomentar las competencias ciudadanas en los estudiantes desde los diversos contextos educativos, para que junto con la formación en valores consigan trabajar en su proyecto de vida". Indican que formar buenos ciudadanos desde la infancia tiene como resultado personas capaces de vivir en comunidad y de desarrollar un proyecto de vida que respete a los demás, acepte diferencias y esté basado en valores de convivencia y esto es posible uniendo proyectos de valores con proyecto de vida.

Finalmente, a pesar de los diferentes puntos de vista que abarcan los estudios expuestos anteriormente, la gran mayoría de estos coinciden en la importancia que tiene el apoyo social en la vida de los adolescentes, ya sea para tomar decisiones, para guiarlos e incluso para prevenir que se expongan a situaciones de riesgo.

De igual forma, como un factor en el desarrollo sano del adolescente, a pesar de encontrarse en un periodo caracterizado por cambios hormonales, emocionales y físicos, la motivación, su deseo

\section{UU


de superación y la comprensión de distintos temas influyen de manera positiva en la formación de metas y proyecto de vida.

Krauskopf (2011), divide la adolescencia en varios periodos psicosociales, entre los cuales menciona el periodo de interés de este estudio como etapa de Adolescencia-Juventud, que abarca de los 17 a los 19 años de edad. Identifica en este periodo una serie de situaciones como ansiedad frente a decisiones que proyectan su futuro, un duelo adolescente y un renacer, un duelo de padres ante el adolescente fantaseado, un cambio en el modelo de autoridad aceptado, una autoridad confusa, una paulatina reestructuración de las relaciones familiares, un desarrollo de instrumentos para la adultez, la exploración de opciones sociales y proyectos, avances y conflictos en la elaboración de la identidad, una creciente orientación al mundo exterior y a la búsqueda de satisfacciones, el encuentro con grupos afines a sus intereses, iniciativas participativas, inicio de relaciones de pareja con diferenciación e intimidad, y la consiguiente capacidad de autocuidado y cuidado mutuo.

Como se puede notar, el propio periodo de adolescencia interfiere en el desarrollo del propio proyecto de vida, sobre todo en lo que respecta a la ansiedad que experimentan hacia el futuro y la construcción de la propia identidad.

Con estos datos pareciera lógico pensar que a los adolescentes les cueste un gran trabajo tomar decisiones que vayan a marcar el resto de sus vidas, como la elección de carrera. Es aquí cuando entra la importancia del apoyo por parte de los padres, profesores y otros allegados que logren servir de guía para ellos tanto educándolos como dándoles contención y apoyo. 


\section{Método}

La investigación realizada es de tipo cuantitativo, los datos fueron recolectados por medio de un instrumento de medición que utilizó escala tipo Likert. El instrumento estuvo conformado por ítems que pretendieron medir distintas variables que fueron de interés para la presente investigación. La información se obtuvo por medio de escalas métricas que permitan una generalización de los resultados.

Se tomaron todos los estudiantes de cuarto año de los colegios Manuel Benavides y Colegio Santa Inés. Participaron 120 estudiantes (61 mujeres, 61 de colegios públicos) con edades entre los 15 y 18 años $(\mathrm{M}=16.40, \mathrm{DE}=.70)$. Tomando en cuenta que se realizó un análisis de regresión múltiple con tres predictores y un tamaño de efecto de .35 , se obtuvo un poder estadístico de .99 .

\section{Resultados}

\section{Correlaciones.}

Al realizar un análisis de correlación simple, se obtuvo que existe una relación positiva entre la variable Proyecto de Vida y las variables Valores $(r=.41 ; p<.001)$, Autoconcepto $(r=.37 ; p<.001)$ y Apoyo Social $(r=.26 ; p=.005)$. Esto quiere decir que a mayor puntuación en estas variables, los adolescentes poseen mayor claridad sobre su Proyecto de Vida. Además se encontró una relación entre Proyecto de Vida y el sexo femenino $(r=-.33 p<.001)$, indicando que las mujeres poseen una mayor claridad respecto a su Proyecto de Vida.

Por otra parte, no se encontró una relación entre Proyecto de Vida y las variables Edad ( $r=$ $.10 p=.257)$ y Tipo de Institución ( $r=.106 p=.248)$.

\section{US


Tabla 1. Medias, desviaciones típicas y correlaciones simples entre las variables en estudio

\begin{tabular}{lll}
\hline Variable & $\alpha$ & Proyecto de Vida \\
\cline { 2 - 3 } Proyecto de Vida & .71 & - \\
Valores & .78 & $.41^{*}$ \\
Autoconcepto & .72 & $.37^{*}$ \\
Apoyo Social & .93 & \\
Sexo & & -.33 \\
Edad & - & $-.10^{*}$ \\
Tipo de Institución & - & $.106^{*}$ \\
\hline
\end{tabular}

* La correlación es significativa al nivel 0,01

\section{Regresiones.}

Se realizó un análisis de regresiones el cual explica un 52\% de varianza $\left(\mathrm{f}_{3,116}=13.99 p<.001\right)$ y se obtiene como principal predictor la variable Autoconcepto ( $\beta .250 p=.005)$ seguido por la variable Valores $(\beta .243 p=.009)$ y por último el Sexo $(\beta .225 p=.009)$ teniendo que a mayor Autoconcepto, más Valores y perteneciendo al sexo femenino más claro es el Proyecto de Vida en los adolescentes.

Tabla 2. Resumen de regresión jerárquica para las variables que pronostican el Proyecto de Vida en adolescentes $(n=120)$.

\begin{tabular}{lll}
\hline Modelo 1 & & \\
\hline $\mathrm{R}_{2}=.173, \mathrm{~F}=24.61, \mathrm{gl}=1$ & & .415 \\
$\mathrm{~B}$ & Valores & \\
\hline Modelo 2 & & .321 \\
$\mathrm{R}_{2}=221, \mathrm{~F}=16.57, \mathrm{gl}=2$ & Valores & .239 \\
$\mathrm{~B}$ & Autoconcepto & \\
& & .243 \\
Modelo 3 & & .250 \\
$\mathrm{R}_{2}=.266, \mathrm{~F}=13.99, \mathrm{gl}=3$ & Valores & -.225 \\
$\mathrm{~B}$ & Autoconcepto \\
& Sexo & \\
\hline
\end{tabular}

${ }^{* *} p<0.001 ;{ }^{*} p<.01$ 


\section{Discusión}

Los resultados obtenidos muestran una comprobación de la hipótesis establecida en esta investigación, es decir, se encontró que existe una relación entre las variables Valores, Autoconcepto y Apoyo Social con respecto al Proyecto de vida, determinando que un mayor puntaje en estas variables permite que los estudiantes posean más claridad con respecto a su Proyecto de Vida. Además se pudo conocer también que el sexo posee relación con la variable principal, arrojando que el sexo femenino suele tener una mayor claridad en este ámbito.

Como se habló anteriormente en la bibliografía estudiada Giménez, Vázquez y Hervás (2010) mencionan la importancia de evitar centrarse en los aspectos negativos de los adolescentes y en su lugar buscar explotar sus potencialidades para que estos tuvieran mayor facilidad para tomar decisiones orientadas hacia el futuro. Según los resultados obtenidos en este estudio, aquellos adolescentes que poseen un mayor conocimiento sobre sus capacidades, quienes se consideran importantes y poseen una posición positiva respecto a sí mismos van a tener una mayor claridad sobre su proyecto de vida.

El estudio de Álvarez et al (s.f.) fue mencionado al inicio de la investigación ya que afirmaba el cómo fomentar el valor de la responsabilidad ayuda a los adolescentes a construir planes para formar su futuro. Además Domínguez (2002) afirma que dentro de la construcción del proyecto de vida intervienen los valores que posee el joven, su concepción del mundo y su identidad personal, datos que coinciden con los resultados obtenidos en este estudio donde se confirma que aquellos adolescentes que consideran importante comportarse según los valores aprendidos poseen mejor claridad en cuanto a la construcción del proyecto de vida.

\section{UT




\section{Vargas}

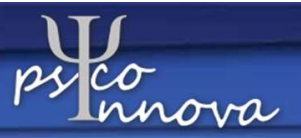

Como se pudo ver en la presente investigación, sentir que se cuenta con personas que brinden apoyo y ayuda ya sea en los momentos buenos como en los malos facilita que los adolescentes piensen su proyecto de Vida con mayor claridad, puesto que un sentido de pertenencia en los adolescentes posee efectos positivos en varias áreas de la vida incluyendo la satisfacción escolar y un estilo de vida más asertivo. En este mismo ámbito Roebken (2007) afirma que la satisfacción escolar permite que los adolescentes estén más dispuestos a alcanzar sus metas.

Tomando en cuenta lo mencionado por Moreira, Sánchez y Mirón (2010) se debe prestar atención a la diferencia por género en cuanto al manejo de relaciones interpersonales, en los resultados obtenidos se pudo observar que las mujeres suelen tener mayor claridad sobre su proyecto de vida, lo cual puede estar relacionado con esta diferencia que mencionan los autores, ya que es más común que las mujeres establezcan relaciones más cercanas con sus pares y les sea más sencillo solicitar apoyo y acompañamiento en sus decisiones, al tiempo que los hombres suelen tener amistades que podríamos considerar más superficiales.

Cabe recordar que uno de los puntos más importantes en la adolescencia es la construcción de la identidad, como menciona Molina (s.f.), puesto que conocer quiénes somos favorece a desarrollar planes de Vida más acertados y para lograr esto las relaciones interpersonales ejercen gran influencia ya que ayuda a diferenciarnos de los demás.

Como mencionan Gómez et al (1998) y también lo encontrado en los resultados de la presente investigación, el Proyecto de Vida y la Autopercepción tiene un estrecha relación, percibirnos de manera positiva nos ayuda a establecer planes y metas a largo plazo, asimismo tener claridad sobre planes a futuro trae consigo beneficios a nuestra autoimagen.

Finalmente, no se demuestra que exista algún tipo de relación entre el tipo de institución a la que asistan los jóvenes y la claridad sobre el proyecto de vida que estos albergan. 


\section{Referencias}

Acosta, D., \& Gastelo, R. (2012). Embarazo en la Adolescencia y Proyecto de vida. Caso: Liceos Públicos de Cumaná, Municipio Sucre, Estado Sucre Año Escolar 2010-2011 (Tesis de Grado). Universidad

de Oriente, $\quad$ Cumaná. $\quad$ Recuperado de http://ri.biblioteca.udo.edu.ve/bitstream/123456789/2327/1/TESIS_DAyRG\%20\%282\%29.pdf

Álvarez, A. (2005). Taller sobre Drogas, Sexualidad y Proyecto de Vida (Tesis de Grado sin publicar). Universidad de Iberoamérica, Costa Rica.

Álvarez, T., Gámez, A., Liébana, L., y Resola, J. (s.f.). Guía de Orientación para Familias con Adolescentes. Consejería de Educación. Delegación provincial de Jaden. Recuperado de http://diversidad.murciaeduca.es/orientamur/gestion/documentos/guia_de_orientacion_para_familia s_con_adolescentes.pdf

Artavia, S. (2011). Relación entre la autoestima y la Elección Vocacional de los estudiantes de quinto año del Instituto Educativo San Gerardo (Tesis de Grado sin publicar). Universidad de Iberoamérica, Costa Rica.

Center for Population Options. (1990). Cómo planear mi vida. Recuperado de http://www.advocatesforyouth.org/storage/advfy/documents/cpmv.pdf

Chaves, R. (2005). Relaciones Interpersonales: En el Contexto Educativo dirigido a los Adolescentes del Colegio Mont Berkeley Intl. en Londres de Montes de Oca (Tesis de Grado sin publicar). Universidad de Iberoamérica, Costa Rica.

Damon, W., Menon, J., \& Cotton, K. (2003). The Development of Purpose During Adolescence. Stanford University, 7(3), 119-128. 
Domínguez, L. (2002). Identidad, Valores y Proyecto de Vida. Revista Cubana de Psicología, 19(2).

Recuperado

de

\section{http://pepsic.bvsalud.org/scielo.php?pid=S025743222002000200009\&script $=$ sci arttext}

Escudero, V. (2011). Adolescentes y familias en conflicto. Terapia familiar centrada en la Alianza

$\begin{array}{lll}\text { Terapéutica. } & \text { Recuperado http://uiicf.net/wp- }\end{array}$

content/uploads/2014/06/ManualAdolescentes_Meninos.pdf

Freite, W., Vesga, I., \& Villanueva, B. (2011). Desarrollo Mi Proyecto De Vida Formándome En Valores Y Demostrando Que Soy Un Buen Ciudadano (Proyecto Transversal). Institución Educativa Distrital

Karl Parrish, Colombia. $\quad$ Recuperado de

http://iedkarlparrish.barranquilla.edu.co/media/Descargas/COMPETENCIAS\%20CIUDADANAS.p

df

Giménez, M., Vázquez, A., y Hervás, G. (2010). El análisis de las fortalezas psicológicas en la adolescencia: Más allá de los modelos de vulnerabilidad. Psychology, Society and Education, 2(2), 97-116.

Gómez, A., Rojas, G., Barquero, M., y Rodríguez, M. (1998). El Proyecto de Vida de los y las adolescentes (Tesis de Grado sin publicar). Universidad de Costa Rica, Costa Rica.

Krauskopf, D. (2011). Etapas Del Desarrollo Psicosocial En La Adolescencia. Clínica Alemana. Recuperado de

http://adolescencia.sochipe.cl/subidos/catalogo3/ETAPAS\%20DESARROLLO\%20PSICOSOCIAL \%20ADOLESCENCIA.pdf

Molina, C. (n.d.). Identidad, identidades y ciencias sociales contemporáneas; conceptos, debates y retos. $\begin{array}{lll}\text { Psicología Online. } & \text { Recuperado }\end{array}$ 
http://www.psicologiaonline.com/articulos/2008/05/identidad_identidades_y_ciencias_sociales.sht $\mathrm{ml}$

Moreira, V., Sánchez, A., y Mirón, L. (2010). El Grupo De Amigos En La Adolescencia. Relación entre afecto, conflicto y conducta desviada. Universidad de Valencia, 100, 7-21.

Mosca, A., y Santiviago, C. (s.f.). Conceptos y herramientas para aportar a la orientación vocacional $\begin{array}{lllll}\text { ocupacional de } & \text { los } & \text { jóvenes. }\end{array}$ http://www.cse.edu.uy/sites/www.cse.edu.uy/files/documentos/MANUAL\%20CONCEPTOS\%20Y \%20HERRAMIENTAS\%20OVO.pdf

Orviz, B., Sánchez, R., \& Valdés, S. (2011). Proyecto de vida a futuro en el adolescente. Centro Cultural $\begin{array}{llll}\text { Itaca } & \text { S.C. } & \text { Recuperado }\end{array}$ http://www.i.edu.mx/aportaciones/Proyecto $\% 20 \mathrm{de} \% 20 v i d a \% 20 \mathrm{a} \% 20$ futuro $\% 20 \mathrm{en} \% 20 \mathrm{el} \% 20$ adoles cente.pdf

Roebken, H. (2007). Multiple goals, satisfaction, and achievement in university undergraduate education: A Student Experience in the Research University (SERU) Project Research Paper. Center for Studies in Higher $\quad$ Education. $\quad$ Recuperado de http://cshe.berkeley.edu/publications/docs/ROP.Roebken.2.07.pdf

Salazar, D. (2009). Planeación de un proyecto de vida: una propuesta para los adolescentes. Universidad Pedagógica Nacional. Recuperado de: http://biblioteca.ajusco.upn.mx/pdf/26576.pdf

Salgado, A. (2009). Felicidad, resiliencia y optimismo en estudiantes de colegios nacionales de la ciudad de Lima. Liberabit, 15(2), 133-141.

Secretaría de educación Jalisco. (2008). Orientación vocacional, proyecto de vida y toma de decisiones en educación secundaria. Dirección de Psicopedagogía. Recuperado de: 


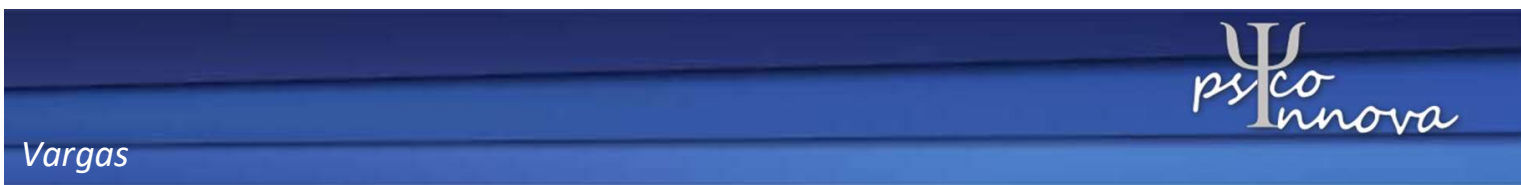

http://portalsej.jalisco.gob.mx/psicopedagogia/sites/portalsej.jalisco.gob.mx.psicopedagogia/files/or ientacion_vocacional_proyecto_vida.pdf

Tesouro, M., Palomares, M., Bonachera, F., y Martínez, L. (2013). Estudio sobre el Desarrollo de la Identidad en la Adolescencia. Tendencias Psicopedagógicas, 21. Recuperado de http://www.tendenciaspedagogicas.com/Articulos/2013_21_15.pdf 